\title{
Neural Fuzzy System for Improved Decision Making For the Selection of Coding Scheme for the On Chip Communication
}

\author{
Dr.M.Jasmin ${ }^{1}$, Dr.D.Gokulakrishnan ${ }^{2}$, Dr.M.Sriram ${ }^{3}$ \\ \{rifriz@gmail.com¹, gokulkrish1453@gmail.com², msr1sriram@gmail.com\} \\ Associate Professor, ECE, Bharath Institute of Higher Education and Research, Chennai, India ${ }^{1}$, \\ Associate Professor, ECE, Sri Sairam Institute of Technology, Chennai, India ${ }^{2}$, \\ Associate Professor, CSE, Bharath Institute of Higher Education and Research, Chennai, India ${ }^{3}$
}

\begin{abstract}
Single coding scheme for the on chip communication in the SOC system may not be sufficient in the heterogeneous on chip communication .It is required to have multiple coding scheme and selection of optimal coding scheme is based on the communicating block. Selection of optimal coding scheme for the on chip communication is important to decide the overall performance. This paper proposes neural fuzzy system based optimal selection scheme to improve the performance . The fuzzy system will be able to provide optimal decision in presence of contradictory constraints and the neural network can able to provide a learning capability for the system .The results shows that the neural fuzzy system is able to provide superior performance comparing to the fuzzy alone system.
\end{abstract}

Keywords: Neural Fuzzy System, SOC system, Optimal Coding, Neural Network.

\section{Introduction}

Due to outstanding system modeling and control capability, neural fuzzy networks have been widely used in the literature. The structure of neural fuzzy networks possesses both the advantages of neural networks and of fuzzy systems. It brings the low-level learning and computational power of neural networks into fuzzy systems and provides the high-level human-like thinking and reasoning of fuzzy systems into neural networks.

Artificial neural fuzzy system is employed to pre distort the OFDM signal such that the output of power amplifier will be exactly the original signal without distortion [1].A neuro fuzzy agent is developed as a reconfigurable system on chip for ambient intelligent application [2]. The work reduced the computational complexity, gives scalability and modularity. Further to reduce the complexity, the dimensionality of the data is reduced by exploitation of redundancy in the data. A nuero fuzzy system that can reduces feature by identifying bad feature is proposed through a mechanism called membership modulator [3].Accurate prediction of wind speed is carried out by using a neural fuzzy system which is able to predict wind speed in hourly basis [4].An engine fault diagnosis is carried out using adaptive neurofuzzy inference system. From the training input and output data, a fuzzy inference system is developed by adjusting the membership function using a least squares method based on hybrid learning algorithm [5]. 
A comparison analysis between support vector machine (SVM) and adaptive neuro-fuzzy inference system (ANFIS) is presented for the classification of electromyography (EMG) signals [6]. The EMG signals were acquired from seven hand common gestures. The ANFIS classification is able to achieve high accuracy than SVM around $91.43 \%$ with reduced feature set by applying principal component analysis (PCA). Brightness adjusting by adaptive neurofuzzy inference system is carried out for improve the object recognition process when improper intensity of light is presented [7].The adaptive neuro-fuzzy inference system is configured as control function to adjust the brightness.

An intelligent sensor for fault detection and compensation (FDC) is proposed for a hybrid grid with renewable energy (RE) sources [8]. The sensors in the control algorithm is crucial one if fault occurs in it which will result in system collapse. This issue is handled by a robust sensor fault control system using ANFIS. A recurrentmethod is provided for filtering the signal noise based on Lyaponov stability which can adaptively filter impulse noise filter. The recurrent method filtering input signal in order to construct ANFIS then that is used as updated-filter to filter the signal noise component [9].

An energy optimization of multi-carrier energy systems is given by combining an adaptive neuro-fuzzy inference system in order model and forecast the power demand of a plant, and a genetic algorithm to optimize the energy flow taking. The optimization algorithm is designed to satisfy the total power demand of the plant and minimizes optimization criteria such as energy usage, monetary cost and environmental cost [10].

The contribution of this work is summarized below:

1. There is no ANFIS system for selection of coding scheme for on chip communication of SOC .So this work will be unique.

2. The proposed ANFIS will be improving our previously developed fuzzy decisive coding scheme [11] by introducing learning capability.

3. The improvement of $10-50 \%$ is observed comparing our previous fuzzy alone system

\section{System model}

The system of neuro fuzzy for the optimal channel coding consists of neural network and fuzzy decisive system. Figure 1 shows the block diagram of cooperative neuro fuzzy system.The neural network is connected in the cooperative mode under the proposal. Under the cooperative mode the neural network operated only once to predict the parameters for the given environmental data. Then the predicted data is feed to the fuzzy unit to make the final decision for the selection of optimal coding scheme. The back propagation neural network architecture is used in this work.

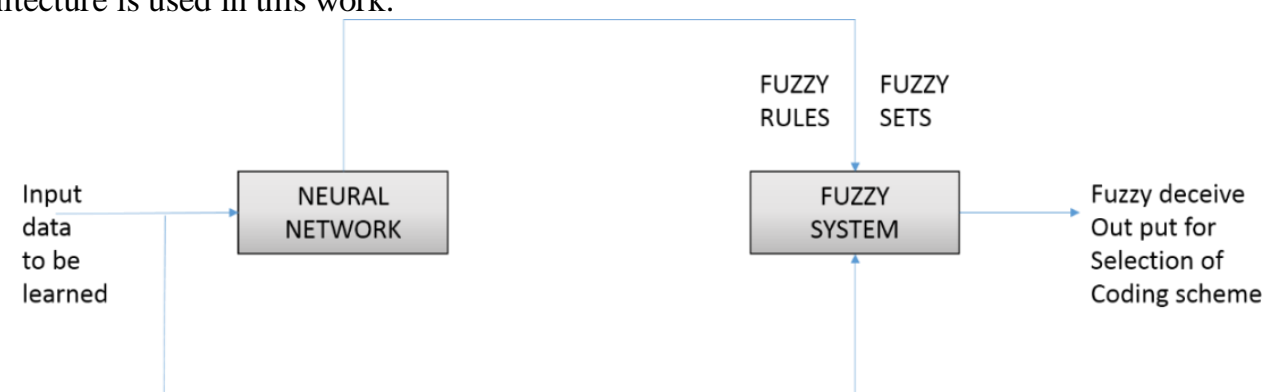

Figure 1.Cooperative Neuro Fuzzy System for Coding Scheme Selection 


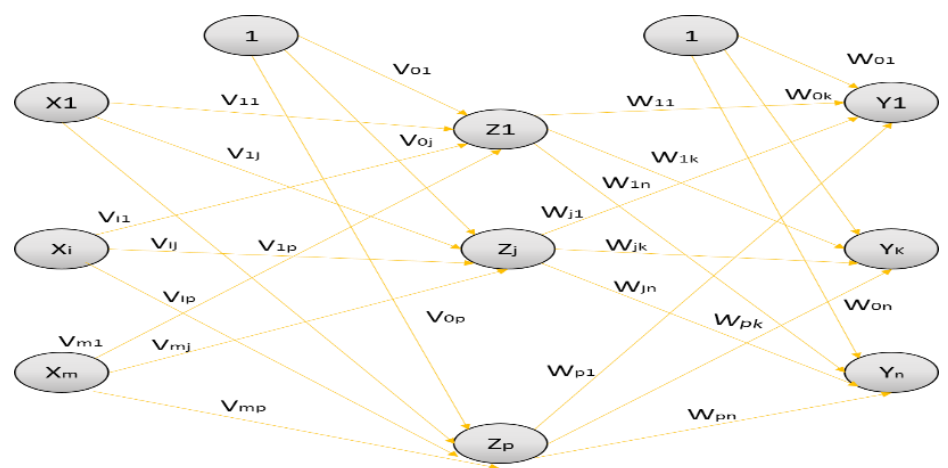

Figure 2.Back Propagation Neural Network Architecture

The architecture of the back propagation is given in figure 2. The architecture consist one input layer, one hidden layer and one output layer .The number of neuron in the input layer is 4 and the number of neuron in hidden layer is 55 and the neuron in output layer 4 .

\section{Result and Discussion}

Random demand of QoS demanding input data's of sample of 1000 is generated which is applied to neural network to train and the trained neural network output is applied to fuzzy system which uses rule based reasoning to select the optimal coding scheme .In order to make different QoS different data types like chaotic random signal, audio signal, image and text are generated and applied to train the neural network and the output of trained neural network is used to adaptively change the parameters of fuzzy parameters and the membership function.

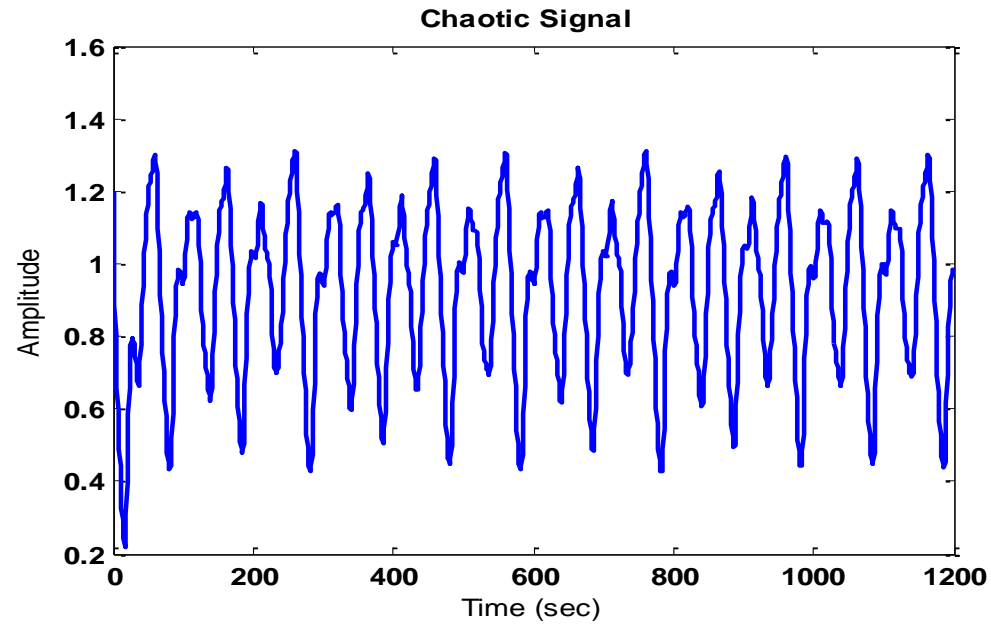

Figure 3.Sample Input Signal of Chaotic Signal

Figure 3 shows the sample input data of chaotic signal (random signal) fed to the neural network section. Using the above signal the neural network is trained which resulted in change of membership function of the fuzzy inference system. After training the reshaped 
membership functions are plotted in figure .4.The reshaped membership function from the trained data ensure that the selection of error control code for the given input data type will be more accurate than that of fuzzy alone system.
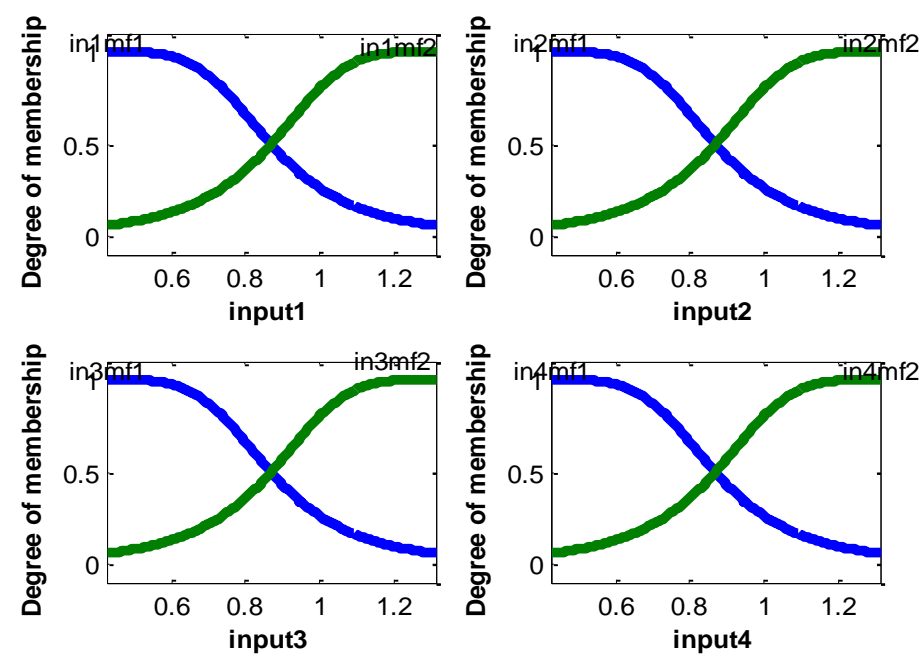

Figure 4.membership function after neural network training

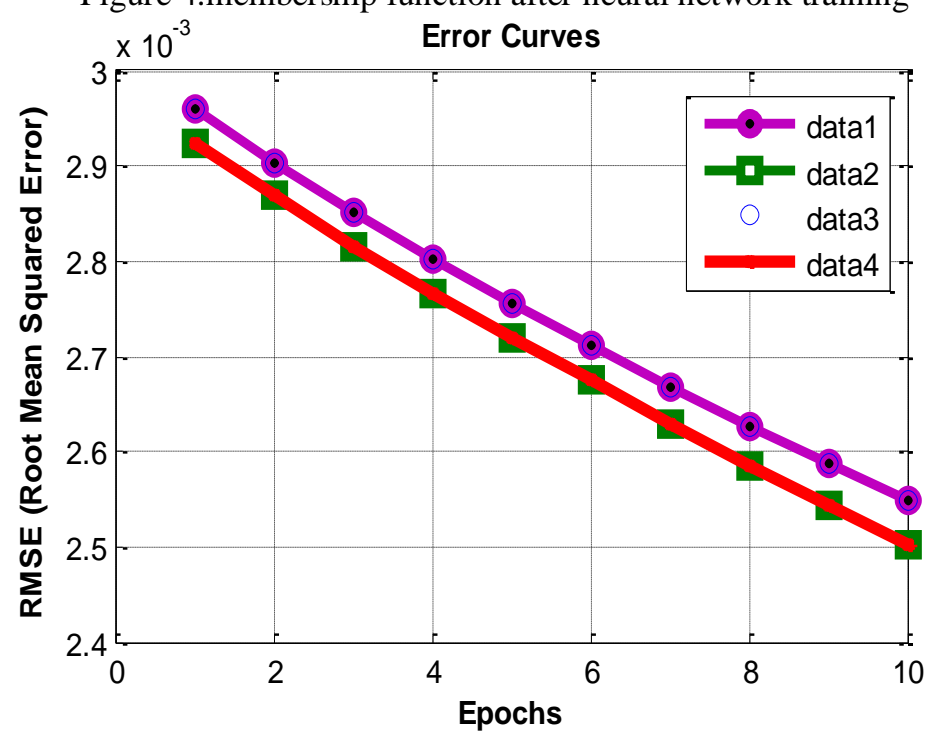

Figure 5.Error values on training and validation

Figure .5 gives the root means square error of the neural network learning process .It can be observed that with respect to increased training steps (Epochs) the root mean square error is getting reduced.It is also observed that for image and video data same error values are taken and for audio and text same error values are taken due to the previous training input.

In order to evaluate the impact of the introduced neural network on the fuzzy system, the error rate of the data transmission of the adaptive coding scheme for the various input data set is evaluated with neural network and without neural network by introducing noise signal into 
the system .Table 1 gives the error rate analysis of the system for the signal to noise ratio on condition of $5 \mathrm{~dB}$.

Table 1.BER improvement of performance

\begin{tabular}{|c|l|l|}
\hline Parameter & Before neural network & After neural network \\
\hline audio & $10^{-3}$ & $0.5 \times 10^{-3}$ \\
\hline Image/video & $10^{-4}$ & $0.6 \times 10^{-4}$ \\
\hline Text & $10^{-5}$ & $0.1 \times 10^{-5}$ \\
\hline $\begin{array}{c}\text { Average computational } \\
\text { time by random type of data } \\
\text { of size } 10 \mathrm{Mb}\end{array}$ & $300 \mathrm{~ms}$ & $220 \mathrm{~ms}$ \\
\hline
\end{tabular}

From the table 1 it can be observed that for audio data $50 \%$ improvement is achieved for the audio data, $40 \%$ improvement is achieved for image and video and $10 \%$ improvement is achieved for the text data type. The table also prove that the average computational time of the adaptive coding scheme is also improved for the random generation of input data type.80ms time is reduced because of selection of low complexity coding scheme on some input data type comparing to that of fuzzy alone system.

Table .2 resource occupancy of coding scheme

\begin{tabular}{|c|c|c|c|c|c|}
\hline scheme & $\begin{array}{l}\text { FPGA } \\
\text { platform }\end{array}$ & $\begin{array}{l}\text { Logic Slice } \\
\text { LUT }\end{array}$ & Registers & Delay & $\begin{array}{l}\text { Power } \\
\text { consumptio } \\
\mathrm{n}\end{array}$ \\
\hline $\begin{array}{l}\text { ANTON, } \\
\text { Constantin[12] } \\
\text { Hamming } \\
\operatorname{code}(15,5)\end{array}$ & $\begin{array}{l}\text { SPARTA } \\
\text { N } 3\end{array}$ & $46 / 3584$ & $58 / 7168$ & $5.778 \mathrm{~ns}$ & $\begin{array}{l}\text { Not } \\
\text { reported }\end{array}$ \\
\hline $\begin{array}{l}\text { Jayan, } \\
\text { Geethu[13]. } \\
\text { Hamming code }\end{array}$ & $\begin{array}{l}\text { SPARTA } \\
\text { N } 3\end{array}$ & $\begin{array}{l}58409.4 \text { micro } \\
\text { Metter }\end{array}$ & $\begin{array}{l}\text { Not } \\
\text { reported }\end{array}$ & $6.7 \mathrm{~ns}$ & $20.5 \mathrm{~mW}$ \\
\hline $\begin{array}{l}\text { Saleh, } \\
\text { H.[14]Hamming } \\
\text { Encoder Design } \\
\text { Status with }(64,7) \\
\text { Code }\end{array}$ & $\begin{array}{l}\text { SPARTA } \\
\text { N } 3\end{array}$ & $\begin{array}{lr}\text { Encoder: } & \text { Slice } \\
28 / 1920 & \\
\text { 4input } & \text { LUT } \\
\text { 50/3840 } & \\
\text { Decoder:135/1920 } \\
\text { 4input } & \text { LUT } \\
245 / 3840 & \end{array}$ & $\begin{array}{l}\text { Not } \\
\text { reported }\end{array}$ & $\begin{array}{l}\text { Not } \\
\text { reported }\end{array}$ & $\begin{array}{l}\text { Not } \\
\text { reported }\end{array}$ \\
\hline $\begin{array}{l}\text { Proposed } \\
\text { Hamming } \\
\text { code }(15,5)\end{array}$ & $\begin{array}{l}\text { SPARTA } \\
\text { N } 3\end{array}$ & $44 / 3584$ & $58 / 7168$ & $5.12 \mathrm{~ns}$ & $19.5 \mathrm{~mW}$ \\
\hline
\end{tabular}

Table 2 shows the comparison of resource occupancy of proposed scheme comparing with the literature .It is evident from the table that our proposed implementation of hamming code is less resource occupancy comparing literature methods. 


\section{Conclusion}

Adaptive error control coding scheme is used in on chip communication in order to handle effectively error rate for different class of input data type like text, audio, video and image. The accuracy of selection decision is improved by means of fuzzy logic based decision system. The fuzzy system is improved by introducing neural network into the system to provide the learning capability which produced a system called Adaptive Neural Fuzzy Inference System (ANFIS).The result of error rate analysis proves that there is an improvement of $10 \%$ (for text data) to $50 \%$ improvement (for audio data) comparing the fuzzy alone system. $80 \mathrm{~ms}$ time saving in computation is achieved because of the introduced learning capability to the fuzzy system.

\section{References}

[1] Jiménez, Víctor P. Gil, et al. "High power amplifier pre-distorter based on neural-fuzzy systems for OFDM signals." IEEE Transactions on Broadcasting 57.1 (2011): 149-158.

[2] del Campo, Inés, et al. "A system-on-chip development of a neuro-fuzzy embedded agent for ambient-intelligence environments." IEEE Transactions on Systems, Man, and Cybernetics, Part B (Cybernetics) 42.2 (2012): 501-512.

[3] Lin, Chin-Teng, et al. "An interval type-2 neural fuzzy system for online system identification and feature elimination." IEEE transactions on neural networks and learning systems 26.7 (2015): 1442-1455.

[4] Li, Chengdong, et al. "Functional-type single-input-rule-modules connected neural fuzzy system for wind speed prediction." IEEE/CAA Journal of AutomaticaSinica 4.4 (2017): 751762.

[5] Chen Qing-Xie,Wu Chun-Fu,"Research on engine fault diagnosis technology based on ANFIS",Chinese Automation Congress (CAC), 2017: 278 - 281.

[6] Caesarendra, Wahyu, TegoehTjahjowidodo, and Daniel Pamungkas. "EMG based classification of hand gestures using PCA and ANFIS." Robotics, Biomimetics, and Intelligent Computational Systems (Robionetics), 2017 International Conference on. IEEE, 2017.

[7] Kim, EunKyeong, et al. "Image brightness adjustment system based on ANFIS by RGB and CIE L* a* b* ." Multisensor Fusion and Integration for Intelligent Systems (MFI), 2017 IEEE International Conference on. IEEE, 2017.

[8] Sowmmiya, Uthayakumar, and Govindarajan Uma. "ANFIS-based sensor fault-tolerant control for hybrid grid." IET Generation, Transmission \& Distribution 12.1 (2017): 31-41.

[9] Nguyen, SyDzung, Seung-Bok Choi, and Tae-Il Seo. "Recurrent Mechanism and Impulse Noise Filter for Establishing ANFIS." IEEE Transactions on Fuzzy Systems (2017).

[10] Kampouropoulos, Konstantinos, et al. "Multiobjective optimization of multi-carrier energy system using a combination of ANFIS and genetic algorithms." IEEE Transactions on Smart Grid (2016).

[11] Anton, Constantin, et al. "Hardware Implementation Of A Single Bit Error Code Correction."UNIVERSITY OF PITESTI - ELECTRONICS AND COMPUTERS SCIENCE, SCIENTIFIC BULLETIN, No. 9, Vol.2, 2009

[12] Jayan, Geethu, and P. P. Pavitha. "FPGA implementation of an efficient router architecture based on DMC." Emerging Technological Trends (ICETT), International Conference on. IEEE, 2016.

[13] Saleh, H. "Design of Hamming Encoder and Decoder Circuits for $(64,7)$ Code and $(128,8)$ Code Using VHDL." Journal of Scientific and Engineering Research 2.1 (2015): 1-15.

[14] Kiran Kumar, T.V.U., Karthik, B., Improving network life time using static cluster routing for wireless sensor networks, Indian Journal of Science and Technology, 2013, 6(SUPPL5), pp. $4642-4647$ 
[15] Thamarai, P., Karthik, B., Kumaran, E.B., Optimizing 2:1 MUX for low power design using adiabatic logic, Middle - East Journal of Scientific Research, 2014, 20(10), pp. 1322-1326 